\title{
Pharmaceutical Counseling Has a Positive Impact on Quality of Life of Hypertension Patients
}

\author{
Gina Aulia ${ }^{1}$, Eli Halimah², Keri Lestari ${ }^{2}$ \\ ${ }^{1}$ Dr. Hasan Sadikin General Hospital, Bandung, West Java, Indonesia \\ ${ }^{2}$ Department of Pharmacology and Clinical Pharmacy, Faculty of Pharmacy, Universitas Padjadjaran, \\ Jatinangor, West Java, Indonesia
}

\begin{abstract}
Pharmacists play a major role in educating patients regarding drug therapy, in order to achieve optimal health outcomes. The aim of this study was to investigate the effect of pharmaceutical counseling on quality of life (QoL) of hypertensive patients with renal impairment at one of public hospital in Bandung, Indonesia. This study used a prospective experimental design with pretest-posttest design for 2 months. Inclusion criteria in this study were hypertensive patients with renal impairment who received antihypertensive drugs, aged $>18$ years, and signed the informed consent. Exclusion criteria were patients who were not able to fill in the questionnaire, patients with end stage renal diseases, diabetes, and pregnant or lactating patients. Pharmaceutical counseling was given during 2 months of the therapy. Pharmaceutical counseling consisted of education on the appropriate use of drugs and disease management. Schedule of their own medication and leaflet containing educational information were given to the subjects. QoL was assessed using a previously validated Mini-Questionnaire of QoL in Arterial Hypertension (MINICHAL). The questionnaire consisted of 16 two dimensions questions, i.e., mental health and somatic manifestation. We found that the mean increase in

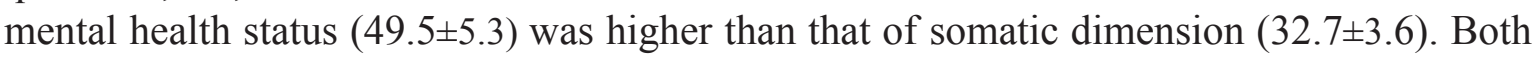
dimensions had statistically significant improvement ( $\mathrm{P}$ value $<0.05$ ). In conclusion, pharmaceutical counseling is beneficial to improve QoL of hypertension patients.
\end{abstract}

Keywords: hypertension, renal impairment, quality of life

\section{Introduction}

Hypertension is one of the leading cause of death, worldwide. It is defined as a systolic blood pressure (BP) $>140 \mathrm{mmHg}$ or a diastolic $\mathrm{BP}>90 \mathrm{mmHg} .{ }^{1}$

The prevalence of hypertension among Indonesian population is $9.5 \% .{ }^{2}$ Uncontrolled blood pressure could lead to impairment of several organs. Renal impairment is one of

Corresponding author: Gina Aulia. Dr. Hasan Sadikin General Hospital, Bandung, West Java, Indonesia. Email:ginaaulia1@gmail.com

Received: 4 March 2018. Revised: 7 April 2018. Published: 23 April 2018. 
the complication that should be carefully monitored. ${ }^{3}$

Barriers to achieve optimal health outcome of antihypertensive therapy include nonadherence to medication and lack of awareness regarding this disease. Pharmacists play an important role in educating the patients regarding drug therapy. Counseling is one of the pharmacist intervention that is expected to improve adherence, knowledge and awareness of patients to the medication, thus improve health outcomes, including quality of life (QoL). QoL is one of the health outcome that could be used as an indicator of the adequate management of the treatment. ${ }^{4,5}$

Several instruments are available to evaluate QoL, including general and disease-related questionnaire. One of the specific questionnaire for hypertension is Mini-Questionnaire of QoL in Arterial Hypertension (MINICHAL). The changes in physical and mental health could be assessed using this instrument. ${ }^{6,7}$

The aim of this study was to determine the effect of pharmacist counseling on the QoL of hypertensive patients with renal impairment.

\section{Methods}

This study used a prospective experimental method with pretest-posttest design. Inclusion criteria in this study were hypertensive patients with renal impairment who received antihypertensive drugs, aged $>18$ years, and signed the informed consent. Exclusion criteria were patients who were not able to fill in the questionnaire, patients with end stage renal diseases, diabetes, and pregnant or lactating patients. This study was conducted at Dr. Hasan Sadikin General Horpial, Bandung, for 2 months period.

Pharmaceutical counseling consisted of education on the appropriate use of drugs and disease management. Schedule of their own medication and leaflet containing educational information were given to the subjects. QoL was assessed using a previously validated Mini-Questionnaire of QoL in Arterial Hypertension (MINICHAL). The questionnaire consisted of 16 two dimensions questions, i.e., mental health and somatic manifestation. The rating scale used in the questionnaire was Likert type scale with four possible answers (0-3).

For mental health dimension, the total score range from 0 (the highest level of health) to 30 (the worst level of health), while for the somatic dimension the score range from 0 (highest health level) to 18 (worst health level). In this study, the score was then transformed into a scale of 100. Statistical analysis was performed using paired t-test with $\mathrm{P}<0.05$ defined statistical significance.

\section{Results and Discussion}

This study included 24 subjects. General characteristics of the subjects can be seen in Table 1. About half (54.2\%) of the subjects are male. Large proportion of the patients aged $>60$ years $(41.7 \%)$. Education of the subjects varied, ranging from elementary school from college education (all categories were below 30\%).

Table 2 illustrates the difference of QoL score, before and after pharmaceutical counseling. In general, we found that mental health score was lower than that of somatic dimensions. Both dimensions scores increased after the completion of the intervention. We found that the mean increase in mental health status (49.5 \pm 5.3$)$ was higher than that of somatic dimension (32.7 \pm 3.6$)$. Both dimensions had statistically significant improvement (P value $<0.05)$. 
Table 1. Characteristics of participants

\begin{tabular}{lcc}
\hline Characteristics & $\mathbf{n}$ & $\mathbf{\%}$ \\
\hline Sex & & \\
$\quad$ Male & 13 & $54.2 \%$ \\
Female & 11 & $45.8 \%$ \\
Age & & \\
$\quad<50$ & 6 & $25.0 \%$ \\
$50-59$ & 8 & $33.3 \%$ \\
$\geq 60$ & 10 & $41.7 \%$ \\
Education & & \\
$\quad$ Elementary school & 5 & $20.8 \%$ \\
Secondary school & 7 & $29.2 \%$ \\
High school & 7 & $29.2 \%$ \\
College & 5 & $20.8 \%$ \\
Occupation & & \\
$\quad$ Worker & 10 & $41.7 \%$ \\
Jobless & 11 & $45.8 \%$ \\
Retire & 3 & $12.5 \%$ \\
\hline
\end{tabular}

Pharmaceutical counseling is effective in modifying beliefs and attitude towards medication, which can result in patients behavioural changes. Such program provides the opportunity for the patients to understand their diseases and clarify misperception regarding the treatment. The elevated $\mathrm{QoL}$ might be resulted from improved awareness of disease and adherence to medication. ${ }^{8,9}$

QoL is a complex concept affecting physical health, psychological conditions, degree of independence, social relationships, individual beliefs and one's relationship to the environment. Nevertheless, the assessment of QoL in hypertension patients is not straightforward since it frequently coexist with other medical conditions, in which QoL can be negatively affected. ${ }^{10}$

Mental health is the most affected dimension among patients with hypertension. Several factors can influenced this finding. Perception of being hypertensive itself might induce pyshiological distress. Besides, the burden of treatment might worsen the mental health. Previous study showed that spirituality helped patients resolving the emotional burden of

Table 2. QoL of participants

\begin{tabular}{lccc}
\hline \multirow{2}{*}{ QoL } & \multicolumn{2}{c}{ Observation } & \multirow{2}{*}{ P-Value } \\
\cline { 2 - 3 } & Before counseling (\%) & After counseling (\%) & \\
\hline Mental Health & $26.2 \pm 12.1$ & $75.7 \pm 6.8$ & $<0.001$ \\
Mean & $6.7-50$ & $56.7-86.7$ & \\
Range & & & $<0.001$ \\
Somatic Health & $49.5 \pm 9.9$ & $82.2 \pm 6.1$ & \\
Mean & $33.3-72.2$ & $72.2-94.4$ & $<0,001$ \\
Range & & & \\
Total & $34.9 \pm 8.4$ & $78.1 \pm 4.8$ & \\
Mean & $18.8-50$ & $64.6-85.4$ & \\
Range & &
\end{tabular}


having hypertension. ${ }^{11-13}$

Our finding is comparable with previous studies showing that pharmacist interventions could improve health-related QoL of hypertensive patients, with or without comorbidity. ${ }^{10,11}$ The strength of our study was the use of specific-disease QoL instruments which could bring more sensitive results for patients with hypertension. The application of this instrument is not only for research purpose, but for clinical application. Data of this study was only obtained from one hospital in Bandung, thus generalizabillity of this finding is limited.

\section{Conclusion}

Pharmaceutical counseling is beneficial to improve QoL of patients with hypertension.

\section{Acknowledgements}

None.

\section{Funding}

None.

\section{Conflict of Interest}

None declared.

\section{References}

1. Egan BM, Zhao Y. Different definitions of prevalent hypertension impact: the clinical epidemiology of hypertension and attainment of healthy people goals. Journal of Clinical Hypertension. 2013;15(3):154-161.

2. World Health Organzation. Global and Regional Review of Hypertension. Avaialble at http://www.searo.who.int/ entity/world_health_day/leaflet_burden hbp_whd2013.pdf?ua $=1$ Accessed $2 \overline{0}$ February 2018.

3. Adeniyi OV, Yogeswaran P, LongoMbenza $\mathrm{B}$, et al. Uncontrolled hypertension and its determinants in patients with concomitant type 2 diabetes mellitus (T2DM) in Rural South Africa. PLoS ONE. 2016;11(3):e0150033.

4. West R, Isom M. Management of patients with hypertension: general practice and community pharmacy working together. The British Journal of General Practice. 2014;64(626):477-478.

5. Soni RK, Porter AC, Lash JP, et al. Healthrelated quality of life in hypertension, chronic kidney disease and coexistent chronic health conditions. Advances in Chronic Kidney Disease. 2010;17(4):1726.

6. Soutello ALS, Rodrigues RCM, Jannuzzi $\mathrm{FF}$, et al. Quality of life on arterial hypertension: validity of known groups of MINICHAL. Arquivos Brasileiros de Cardiologia. 2015;104(4):299-307.

7. Carvalho MAN, Silva IBS, Ramos SBP, et al. Quality of life of hypertensive patients and comparison of two Instruments of HRQOL measure. Arquivos Brasileiros de Cardiologia. 2012;98(5):442-451.

8. Saleem F, Hassali MA, Shafie AA, et al. Pharmacist intervention in improving hypertensionrelated knowledge, treatment medication adherence and health related quality of life: a non-clinical randomized controlled trial. International Journal of Public Participation in Health Care and Health Policy. 2015;18(5):1270-1281.

9. Morgado M, Rolo S, Castelo-Branco M. Pharmacist intervention program to enhance hypertension control: a randomised controlled trial. International Journal of Clinical Pharmacy. 2011;33(1):132-140.

10. Kretchy IA, Owusu-Daaku FT, Danquah SA. Mental health in hypertension: assessing symptoms of anxiety, depression and stress on anti-hypertensive medication adherence. International Journal of Mental Health Systems. 2014;8:25. 
11. Hamer M, Batty GD, Stamatakis E, et al. Hypertension awareness and psychological distress. Hypertension. 2010;56(3):547-550.

12. DeJean D, Giacomini M, Vanstone M, et al. Patient experiences of depression and anxiety with chronic disease: a systematic review and qualitative meta-synthesis. Ontario Health Technology Assessment Service. 2013;13(16):1-33

13. Kretchy I, Owusu-Daaku F, Danquah S. Spiritual and religious beliefs: do they matter in the medication adherence behaviour of hypertensive patients? Biopsychosocial Medicine. 2013;7:15. 